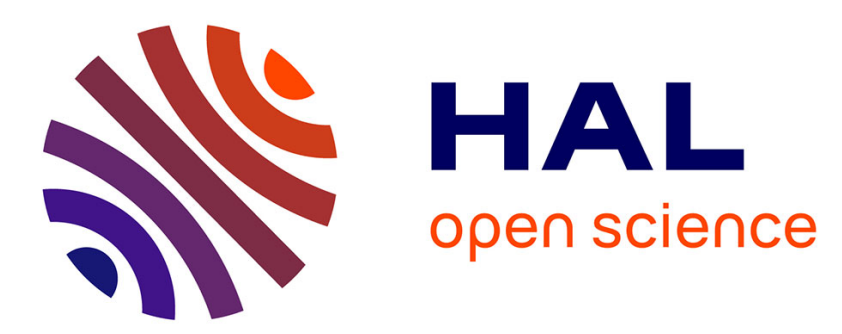

\title{
Design and Analysis of Virtualization Framework for Wireless Sensor Networks \\ Imran Khan
}

\section{To cite this version:}

Imran Khan. Design and Analysis of Virtualization Framework for Wireless Sensor Networks. 5th IEEE International Symposium on a World of Wireless, Mobile and Multimedia Networks PhD forum 2013 (IEEE WoWMoM 2013 PhD forum), 2013, Jun 2013, Madrid, Spain. pp.1-2. hal-00848255

\section{HAL Id: hal-00848255 \\ https://hal.science/hal-00848255}

Submitted on 25 Jul 2013

HAL is a multi-disciplinary open access archive for the deposit and dissemination of scientific research documents, whether they are published or not. The documents may come from teaching and research institutions in France or abroad, or from public or private research centers.
L'archive ouverte pluridisciplinaire HAL, est destinée au dépôt et à la diffusion de documents scientifiques de niveau recherche, publiés ou non, émanant des établissements d'enseignement et de recherche français ou étrangers, des laboratoires publics ou privés. 


\title{
Design and Analysis of Virtualization Framework for Wireless Sensor Networks
}

\author{
Imran Khan \\ Institut Minés-Télécom, Télécom SudParis, \\ 91011 Evry Cedex, France \\ imran@ieee.org
}

\begin{abstract}
Wireless Sensor Networks (WSNs) are used in many application areas including health, agriculture and gaming. New advances in sensor technology make it pertinent to consider sharing a deployed WSN infrastructure by multiple applications, including applications which are designed after the WSN deployment. For my $\mathrm{PhD}$ research I propose a novel WSN virtualization framework that allows multiple users to run their application tasks over underlying WSN resources in a transparent way. This paper presents the overview of the proposed WSN virtualization framework, related work, current status and future work.
\end{abstract}

Keywords-Wireless Sensor Networks; Virtualization; Overlay Networks; Wireless Sensor Network Virtualization;

\section{INTRODUCTION}

Wireless Sensor Networks (WSNs) are combinations of micro-electro-mechanical systems, wireless communication systems and digital electronics nodes that sense, compute and communicate [1]. Up till now the real world deployments of WSNs have been tailor-made solutions where applications are bundled with a WSN at the time of deployment with no possibility for other applications to re-use the deployed WSN. Virtualization is a technique that presents physical resources logically and enables their sharing and efficient usage [2]. The new generations of sensors [3] encourage us to consider sharing them using virtualization.

WSN virtualization is a relatively new field and to the best of our knowledge there is no mechanism to discover and publish WSN resources for multiple, independent applications allowing them to access these resources concurrently according to their requirements. Also there are no WSN oriented protocols for signaling, resource reservation and management for this purpose. Overlays can be used to have multiple applications access the WSN resources but they require careful analysis because of the inherit WSN constraints.

The main contribution of this $\mathrm{PhD}$ work is to provide a platform independent WSN virtualization framework consisting of $i$ ) a general architecture for WSN virtualization, ii) a dynamic discovery and publication framework, iii) a middleware independent overlay protocol suitable for resource constraint WSN nodes, and $i v$ ) a resource efficient signaling protocol for resource reservation and session management. None of the existing works deals with these issues.

The rest of the paper is organized as follows. Related work is presented in Section II. Overview of the proposed WSN virtualization framework is presented in Section III. Current status and future directions are discussed in Section IV and Section V concludes the paper.

\section{RELATED WORK}

In literature, the existing WSN virtualization solutions can be classified into three categories, node level virtualization [4], [5] network level virtualization [6] and hybrid solutions [7], [8]. Our work considers both node and network level virtualization, hence it is pertinent to compare it to the hybrid solutions only. The authors in [7] provide a platform dependent solution for WSN virtualization. The proposed solution works on specific OS and hardware. Each application program uses a hardware abstraction layer (HAL) to access underlying WSN resources, which means that the developer needs to be aware of the HAL, which in turn depends on the OS. The solution in [8] is one of the pioneering work but falls short of true WSN virtualization philosophy. The applications are preconfigured and decided before the deployment of the WSN. It is not possible to include new applications afterwards. In [9] a software architecture FLEXOR is presented which provides optimal implementation, and evaluation of protocols with focus on reusability, QoE and user friendliness in WSN development cycle. This platform, however, does not discuss WSN virtualization. There is no discussion on how multiple applications, developed using FLEXOR, will use the underlying WSN resources concurrently.

\section{WSN VIRTUALIZATION FRAMEWORK}

\section{A. Basic Principles}

The first principle for WSN virtualization is that any new application or a service is deployed as a new overlay on top of the physical WSN. The second principle is that any given physical sensor can execute (locally) task(s) for a given application deployed in the overlay. Existing sensor kits such as Java SunSpot [3] and operating systems like Contiki [10] support concurrent execution of multiple applications. The third and final principle is that some sensor may not have enough capabilities to support the overlay middleware. When this is the case, they will delegate such operations to more powerful sensors and even to other nodes.

\section{B. Proposed WSN Virtualization Framework}

Figure 1 shows the proposed WSN virtualization framework. In this framework heterogeneous sensor nodes with varying 


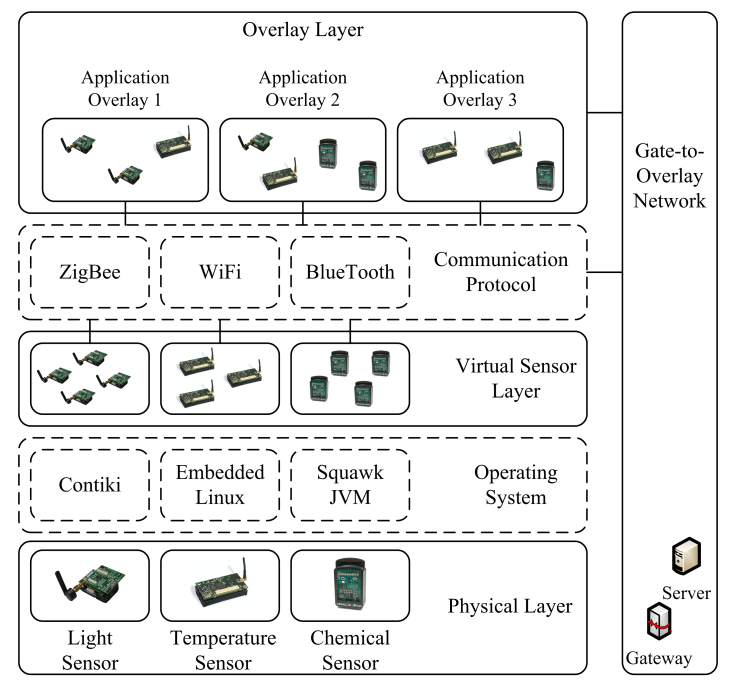

Fig. 1. WSN Virtualization Framework

sensing capabilities are considered. All sensor nodes have an OS that supports concurrent execution of the application tasks and can communicate using variety of communication protocols. There is a virtual sensor layer that consists of logical instances of sensors executing distinct application tasks. E.g. if a sensor executes two application tasks, it will have two virtual sensors each dedicated to a single task. The number of virtual sensors supported by a sensor node depends on its resources.

The end user applications create application specific overlays. The virtual sensors become part of these overlays as overlay nodes and participate in the execution of the end user applications as per the first principle of the framework. The creation of virtual sensors is according to the second principle. As for the final principle, there are certain nodes (e.g. gateways or servers) that enable resource deficient sensor nodes to join the application overlays. Such nodes are termed as Gate-toOverlay (GTO) nodes making up a GTO network helping virtual sensors to join /leave application overlays.

\section{Current Status And Future Directions}

A multi-layer general architecture for WSN virtualization is presented in [11]. At present an initial prototype is under development using Java SunSpot kit and JXTA middleware. Depending on the progress and acceptance of this extended abstract, initial results will be presented at the conference.

For future work, identified research issues include, a publication and discovery framework to allow different actors, including sensors, to publish and discover on the fly. This includes discovery of suitable sensors by the end user applications and discovery of GTO nodes by sensors to participate in the application overlays.

The second issue is to find suitable overlay protocols, especially as these protocols should be middleware-independent whenever possible. Another issue is to manage and prevent overlays from interacting in a harmful way as they compete for the underlying resources (WSN nodes in this case).
The third and final issue is regarding the signaling framework. There are several signaling frameworks like SIP/RSVP but they may not be suitable for resource-constrained devices. A CoAP [12] based signaling framework is a potential solution.

\section{CONCLUSION}

In this paper an overview of WSN virtualization framework and its related issues are presented and some key research issues are also identified. After proposing general architecture, currently work on initial prototype is in progress. WSN virtualization is an emerging area of research that can potentially help to realize the true potential of sensors. Much of this depends on the advancements in the sensor hardware technology but presently we have, in our hands, some capable devices that can be used to initiate research activities in this area.

\section{ACKNOWLEDGMENT}

This work is supervised by Prof. Noel Crespi, Institut MinesTélécom, Télécom SudParis, France and Prof. Roch Glitho, CIISE, Concordia University, Canada. The author would like to thank Dr. Fatna Belqasmi for her comments.

\section{REFERENCES}

[1] Akyildiz, Ian F., et al. "Wireless sensor networks: a survey.", Computer networks 38.4 (2002): 393-422.

[2] S. Loveland, et.al, "Leveraging virtualization to optimize highavailability system configurations", IBM Systems Journal, vol. 47, no.4, 2008. 591-604.

[3] Smith, Randall B. "SPOTWorld and the Sun SPOT", Information Processing in Sensor Networks, 2007. IPSN 2007. 6th International Symposium on. IEEE, Cambridge, MA, 2007. 565-566.

[4] P. Levis and D. Culler: "Maté: A tiny virtual machine for sensor networks", In ASPLOSX: Proceedings of the 10th International Conference on Architectural Support for Programming Languages and Operating Systems, San Jose, CA, 2002, pp. 85-95.

[5] M. Navarro et al., "VITRO Architecture: Bringing Virtualization to WSN World", Mobile Ad-Hoc and Sensor Systems, IEEE 8th International Conference on, Valencia, Spain, 2011, pp. 831-836

[6] A. P. Jayasumana, et al., "Virtual sensor networks a resource efficient approach for concurrent applications", In Proc. Information Technology, 2007. ITNG'07. Fourth International Conference on, Las Vegas, 2007, pp. $111-115$

[7] Leontiadis, Ilias, et al. "SenShare: transforming sensor networks into multi-application sensing infrastructures", Wireless Sensor Networks, Springer Berlin Heidelberg, 2012, pp. 65-81.

[8] Y. Yu et al., "Supporting concurrent applications in wireless sensor networks", 4th International Conference on Embedded Networked Sensor Systems, SenSys06, Boulder, Colorado, 2006, pp.139-152

[9] Forster, Anna, et al. "Flexor: User friendly wireless sensor network development and deployment", World of Wireless, Mobile and Multimedia Networks (WoWMoM), 2012 IEEE International Symposium on a. IEEE, San Francisco, CA, 2012, pp. 1-9

[10] Dunkels, Adam et al., "Contiki-a lightweight and flexible operating system for tiny networked sensors", Local Computer Networks, 2004. 29th Annual IEEE International Conference on. IEEE, Tempa, FL, 2004, pp. 455-462.

[11] Khan, Imran, et al., "A Multi-Layer Architecture for Wireless Sensor Network Virtualization", in 6th Joint IFIP Wireless and Mobile Networking Conference (WMNC'13), April, 23-25, Dubai, UAE, 2013. To appear.

[12] Shelby, et al., "Constraint Application Protocol (CoAP), IETF, InternetDraft, draft-ietf-core-coap-13.txt (work in progress), 2012 